\title{
Production of Human CNS Neurons from Embryonal Carcinoma Cells Using a Cell Aggregation Method
}

BioTechniques 26:946-954 (May 1999)

\author{
William M.W. Cheung, Wing \\ Y. Fu, Wing S. Hui and Nancy \\ Y.Ip \\ Hong Kong University of \\ Science and Technology, \\ Hong Kong, China
}

\begin{abstract}
When treated with retinoic acid (RA), a human embryonal carcinoma (EC) cell line, NTera 2 cl.D/1 (NT2), differentiates into several morphologically distinct cell types, which include terminally differentiated postmitotic central nervous system (CNS) neurons. Accumulating evidence has demonstrated the significant potential of NT2 cells in studies related to cancer therapy and neurodegenerative diseases. However, preparation of enriched NT2 neurons often requires a lengthy period (ca. five weeks) and depends largely on tedious techniques similar to those used for primary neuronal cultures. Here, we report a rapid protocol for the preparation of these human CNS neurons. Using the method of cell aggregation, enriched NT2 neurons can be obtained in approximately two weeks. We also demonstrated that cell aggregation reduced the time normally required for the induction of neuronal differentiation, as revealed by the early expression of neuronal markers. The period of RA treatment could also be reduced if NT2 cells were maintained as aggregates for a sufficient period of time. Taken together, our findings demonstrated that cell aggregation promoted $R A$-induced neuronal differentiation of NT2 cells and provided a rapid protocol for the efficient production of NT2 neurons. The ability to produce large quantities of human CNS neurons should facilitate future use of these neurons for basic research and applications in cell therapy.
\end{abstract}

\section{INTRODUCTION}

Retinoic acid (RA), a derivative of vitamin $\mathrm{A}$, induces differentiation in many cell types, such as epithelial and cancer cells $(4,8)$. Among the human cell types known to respond to RA, the human embryonal carcinoma (EC) cell line, NTera2 cl.D/1 (NT2), is one of the most extensively characterized $(2,16)$. Following treatment with RA for four weeks, NT2 cells, as monolayer cultures, undergo terminal differentiation and become postmitotic central nervous system (CNS) neurons (1). Successive replating of RA-treated NT2 cells, in the presence of growth inhibitors, results in the isolation of purified neurons (NT2-N) (17). These NT2-N neurons have been used as model systems for the study of neurodegenerative diseases such as Alzheimer's disease (7). The potential applications of NT2-N neurons in cell transplantation therapy for CNS disorders, and their use as vehicles for delivering exogenous proteins into the human brain for gene therapy, were recently demonstrated (20).

When compared with murine EC cells such as P19, which are cultured as aggregates and are capable of differentiating into CNS neurons $(3,12)$, NT2 cells require a relatively high concentration of RA $(10 \mu \mathrm{M})$ to induce differentiation. Furthermore, the purification of NT2-N neurons involves a time-consuming process (ca. eight weeks) and tedious primary culture techniques. The procedures of frequent dislodgment and replating might also result in the loss of neuron subtypes, such as those expressing tyrosine hydroxylase (TH), which are found in newly differentiated NT2-N neurons (10). It is possible that the high concentration of RA and the lengthy period required for the differentiation of NT2 cells might partly be explained by the species difference between the two embryonal carcinoma cell lines, i.e., human vs. murine. Alternatively, there exists the possibility that other extrinsic factors, such as cell/cell interaction, might play a critical role in the process of neuronal differentiation in NT2 cells.

The importance of cell aggregation in initiating neuronal differentiation has been reported in other cell types, such as embryonic stem cells and the P19 EC cells $(13,18)$. Because undifferentiated NT2 cells resemble neuroepithelial cells and retain properties of stem cells (16), it is possible that cell/cell interaction can be enhanced by forming cell aggregates as in the P19 cells (11). In the present study, we have examined whether cell aggregation can promote the RA-induced neuronal differentiation of NT2 cells. Here, we report the development of a rapid protocol involving cell aggregation that enhances the process of neuronal differentiation. This provides an efficient methodology for the preparation of human CNS neurons and also allows for a better understanding of the importance of cell/cell interactions, which act in concert with RA to enhance the neuronal differentiation of precursor cells.

\section{MATERIALS AND METHODS}

\section{Cell Culture}

NT2 cells were cultured as previously described (17). Briefly, cells were maintained in OPTIMEM ${ }^{\mathrm{TM}}$ I Reduced Serum Medium supplemented with 5\% fetal bovine serum (FBS; both from 
Life Technologies, Gaithersburg, MD, USA). When NT2 cells were differentiated by the standard protocol, NT 2 cells were seeded at $2 \times 10^{6}$ cells $/ 75 \mathrm{~cm}^{2}$ in Dulbecco's modified Eagle medium (high-glucose formulation) (DMEMHG; Life Technologies) and were treated with a final concentration of $10 \mu \mathrm{M}$ all trans-RA (Sigma, St. Louis, MO, USA). In the cell aggregation studies, cells were dislodged by trypsinization and were plated at $1 \times 10^{5}$ cells $/ \mathrm{mL}$ into a $85-\mathrm{mm}$ bacteriological grade petri dish in DMEM-HG supplemented $10 \%$ FBS. After incubation overnight at $37^{\circ} \mathrm{C}$ in a $5 \% \mathrm{CO}_{2}$ incubator, the aggregates were supplemented with a final concentration of $10 \mu \mathrm{M}$ all trans-RA. The medium was replaced every two days. After 14 days of RA treatment, aggregates were plated onto tissue culture grade petri dishes, pre-coated with $10 \mu \mathrm{g} / \mathrm{mL}$ poly-D-lysine (PDL; Sigma), $10 \mu \mathrm{g} / \mathrm{mL}$ mouse laminin (LAM; Life Technologies) and $0.1 \%$ gelatin (Sigma) in Dulbecco's phosphate-buffered saline (D-PBS; Life Technologies). Following overnight incubation, aggregates were then cultured in DMEM-HG with $10 \%$ FBS supplemented with $1 \mu \mathrm{M}$ cytosine D-arabinofuranoside (araC) and $10 \mu \mathrm{M}$ uridine (both from Sigma). After 3 days of incubation, neurons were detached by tapping gently on the side of the tissue culture plate, or by brief trypsinization and then replated onto a new pre-coated tissue culture plate. Incubation was continued in the presence of araC and uridine for another 4 days. Neurons obtained could be kept in DMEM-HG supplemented with $10 \%$ FBS for more than 3 weeks.

\section{Immunofluorescent Analysis}

For immunofluorescent analysis, control or differentiated aggregate cultures were fixed with $4 \%$ paraformaldehyde (Fluka Chemical, Milwaukee, WI, USA) in PBS. After blocking with $4 \%$ normal serum (NS; Vector Laboratories, Burlingame, CA, USA) in PBS containing $0.4 \%$ Triton ${ }^{\circledR} \mathrm{X}-100$ (TX) and $1 \%$ bovine serum albumin (BSA; Sigma), aggregates were incubated overnight at $4^{\circ} \mathrm{C}$ with primary antibodies diluted in antibody diluent (1\% NS, $0.4 \% \mathrm{TX}$ and $1 \%$ BSA). Incubation with secondary antibodies was per- formed at room temperature for at least $2 \mathrm{~h}$. Cells were visualized using a Zeiss Axiophot ${ }^{\circledR}$ Fluorescence Microscope (Carl Zeiss GmbH, Jena, Germany). Antibodies used for the studies include polyclonal anti-rabbit 200-kDa neurofilament protein (NF-H; Chemicon, Temecula, CA, USA), monoclonal mouse anti-phosphorylated NF-M protein (NF-M-P; Zymed Laboratories, South San Francisco, CA, USA), polyclonal rabbit anti-substance $\mathrm{P}$ (SP; Chemicon), polyclonal rabbit anti- $\gamma$ amino butyric acid (GABA) (Sigma) and polyclonal rabbit anti-tyrosine hydroxylase (TH; Chemicon). Mouse synapsin I antibodies (a generous gift from A. Baines) were used to detect presynaptic specialization. Fluorescein isothiocyanate (FITC)-conjugated goat anti-rabbit IgG (Cappel, Turnhout, Bel-

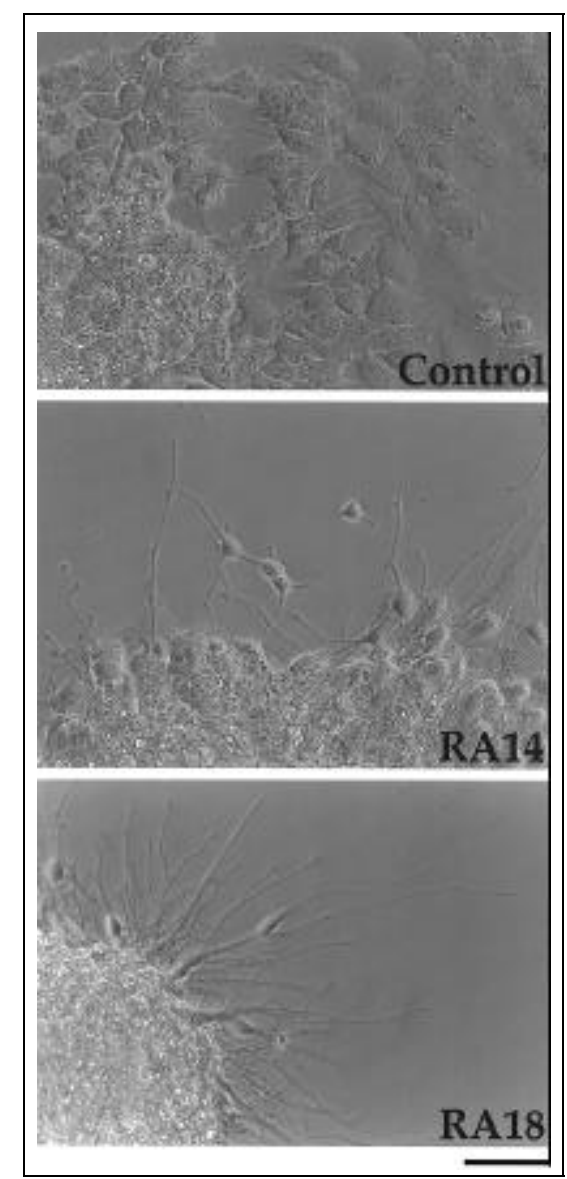

Figure 1. Aggregation promotes the neuronal differentiation of NT2 cells. Cells were pre-aggregated overnight and then cultured as aggregates with RA for 14 (RA14) or 18 (RA18) days. Control cells were maintained as aggregates without RA for 14 days. The bright-field, phase-contrast photomicrographs are shown. Scale bar, $100 \mu \mathrm{m}$. gium) and rhodamine-conjugated goat anti-mouse IgG (Cappel) were used as secondary antibodies.

\section{Total Protein Extraction and Western Blot Analysis}

Cells were washed twice with icecold PBS followed by a wash with icecold PBS supplemented with $1 \mathrm{mM}$ sodium orthovanadate (NaOV; Sigma). Cells were then lysed with lysis buffer [50 mM Tris- $\mathrm{HCl}$ at $\mathrm{pH} 8.0,150 \mathrm{mM}$ $\mathrm{NaCl}, 1 \% \mathrm{TX}, 1 \times$ aprotinin (Sigma), 1 $\mathrm{mM}$ phenylmethylsulfonyl fluoride (PMSF; Life Technologies) and $1 \mathrm{mM}$ $\mathrm{NaOV}]$ at $4^{\circ} \mathrm{C}$ for $15 \mathrm{~min}$. Lysates were collected and centrifuged to remove cell debris. Protein assays were performed with a Bio-Rad Protein Assay Kit based on the Bradford dye-binding procedure (Bio-Rad, Hercules, CA, USA). Protein (typically $10 \mu \mathrm{g}$ ) were separated by sodium dodecyl sulfate polyacrylamide gel electrophoresis (SDS-PAGE) and then electro-transferred to nitrocellulose membrane (Micron Separations Inc. [MSI], Westborough, MA, USA). After blocking at room temperature for $1 \mathrm{~h}$ using Tris-buffered saline with Tween ${ }^{\circledR}$ 20 (TBST) containing 5\% nonfat milk, membranes were incubated overnight at $4^{\circ} \mathrm{C}$ with primary antibodies in TBST containing $5 \%$ nonfat milk. Then, membranes were incubated with horseradish peroxidase conjugated secondary antibodies (1:2000 dilution; Amersham Pharmacia Biotech, Piscataway, NJ, USA) for $1 \mathrm{~h}$ at room temperature. After three washes with TBST, immunoreactive bands were detected using ECL $^{\mathrm{TM}}$ Western Blotting Reagents (Amersham Pharmacia Biotech).

\section{RESULTS}

\section{Cell Aggregation Reduced the Time Required to Form NT2 Neurons}

To examine the effect of cell aggregation on RA-induced neuronal differentiation, NT2 cells were pre-aggregated as described in Materials and Methods. After 14 days of RA treatment, aggregates were plated on PDL and LAM (PDL/LAM)-coated plates. Neurite outgrowth was initiated after overnight incubation in the absence of 


\section{Research Reports}

RA. Phase-bright, neuron-like cells with long, extended neurites continued to migrate out from the NT2 aggregates. At day 3 after replating, these cells grew on the surface of large, flattened cells (Figure 1). If NT2 aggregates were treated with RA for only 12 days, the extension of neurites from NT2 cells was observed 2 days after being plated onto PDL/LAM-coated plates. However, neuron-like cells were subsequently overwhelmed by both undifferentiated NT2 cells and the differentiated large, flattened cells (17). Further reduction of the cell aggregation period to less than 12 days did not result in observable neuritic outgrowth of RA-treated aggregates.

\section{Phase-Bright, Neuron-Like Cells Express Neuronal Markers}

To verify whether the phase-bright cells obtained following cell aggregation were neurons, immunocytochemical analysis was performed. High-molecular-weight neurofilament proteins $(200 \mathrm{kDa})$ were detected in the neurites of the neurons (Figure 2). Presynaptic specialization of the differentiated NT2 neurons was demonstrated by positive staining of the neuron-specific synapsin $\mathrm{I}$ in the varicosities of the neurites and in the cell body (Figure 2). We have also examined the expression of neurotransmitters, such as GABA. Detection of GABA in these neurons (Figure 2) demonstrated the presence of GABAergic neurons in the differentiated NT2 aggregates. In addition, SP was detected in both the cell bodies and the neurites of the neurons (Figure 2). TH-expressing neurons were also found in enriched NT2 neurons prepared using the cell aggregation method (Figure 3 ).

\section{Effect of Substrates on the Extension of Neurites from Differentiated Aggregates}

Neuronal differentiation of NT2 cells maintained as monolayer cultures were previously demonstrated to be influenced by the types of substrates (16). We have compared the effects of various extracellular matrix on the differentiation of NT2 aggregates. After 14 days of RA treatment, NT2 aggregates were plated onto tissue culture plates coated either with gelatin, PDL, LAM alone or in combination (Figure 4). Although gelatin and PDL allowed better attachment of the aggregates onto the plates, the use of PDL alone or PDL in combination with gelatin as substrates did not result in the extension of neurites, even after incubation of NT2 cells for more than 1 week. The presence of LAM alone or in combination with gelatin and PDL resulted in the extension of neurites. Thus, the extension of neurites from NT2 aggregates was dependent on the extracellular substrates.

\section{Neuronal Differentiation of NT2 Cells in Aggregates was Observed at Lower RA Concentrations}

To study whether the high concentrations of RA required for inducing neuronal differentiation of NT2 cells could be lowered under the condition of cell aggregation, we exposed the NT2 aggregates to reduced concentration of RA $(0.1-10 \mu \mathrm{M})$. Following treatment of NT2 aggregates with RA for 14 days, these cells were plated onto PDL/LAM-coated plates in RA-free medium, and total protein samples were prepared 2 days later. As a control, total protein samples were extracted from monolayer NT2 cultures treated with 16 days of RA $(0.1-10 \mu \mathrm{M})$.
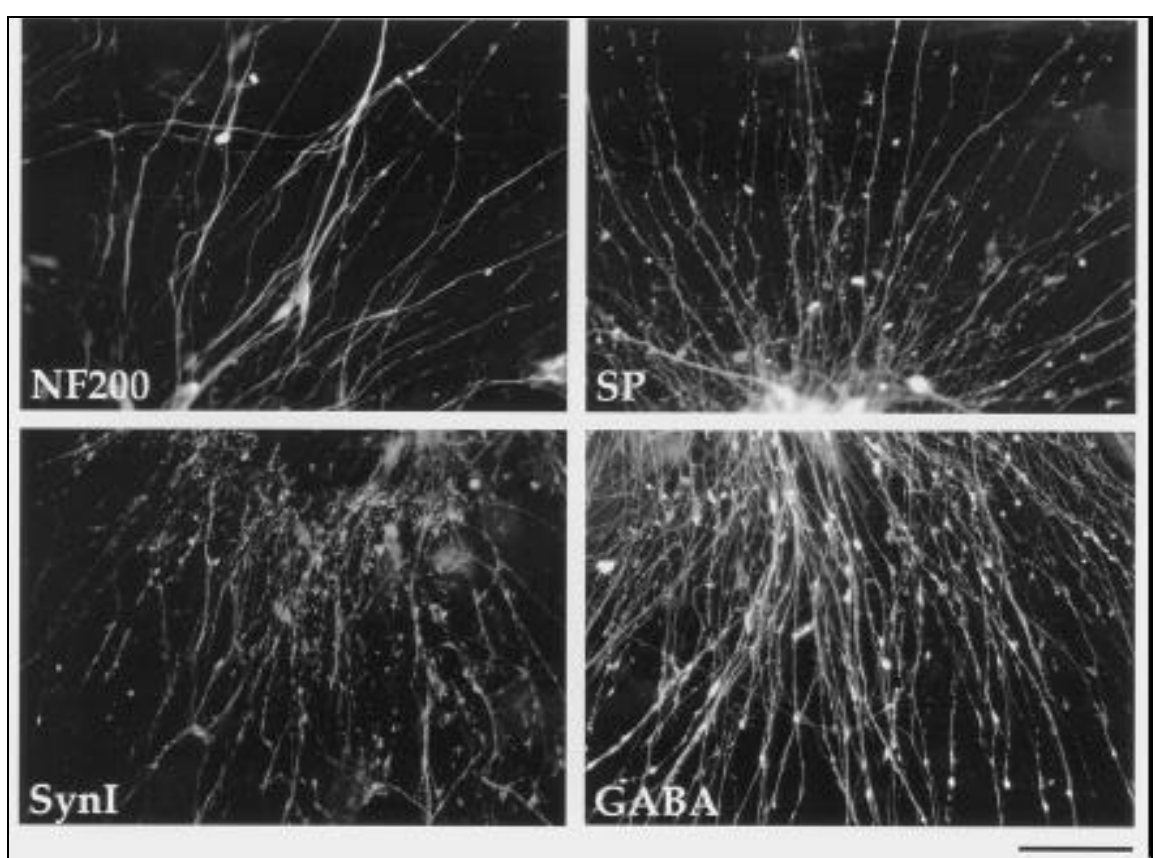

Figure 2. NT2 neurons differentiated by cell aggregation expressed neuronal-specific proteins. NT2 cells were aggregated and differentiated by RA $(5 \mu \mathrm{M})$ for 14 days. Two days after replating and being cultured in RA-free medium, differentiated NT2 cells were fixed and stained with the antibodies specific for NF $200 \mathrm{kDa}$ (NF200), synapsin I (synI), SP and GABA. The bright-field, phase-contrast photomicrographs are shown. Scale bar, $100 \mu \mathrm{m}$.

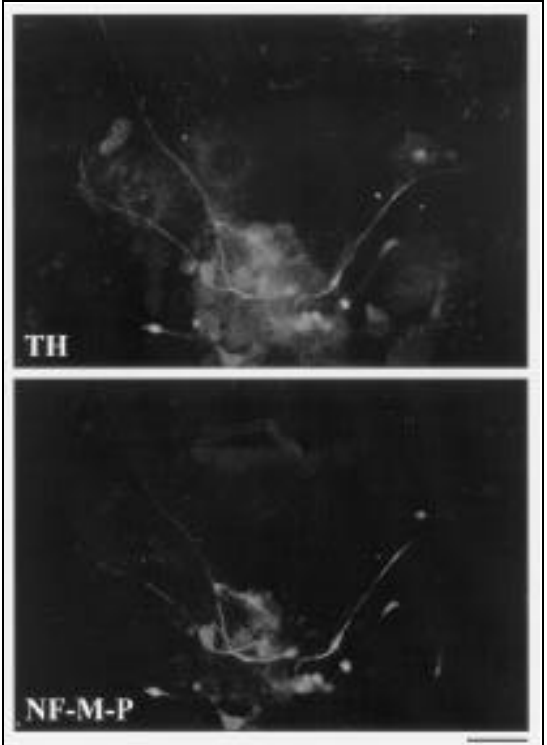

Figure 3. NT2 neurons differentiated by cell aggregation expressed TH. NT2 cells were aggregated and differentiated by RA $(5 \mu \mathrm{M})$ for 14 days. Two days after replating in RA-free medium, differentiated NT2 cells were fixed and stained with the antibodies specific for TH (upper panel) and phosphorylated NF-M (lower panel). Scale bar, $50 \mu \mathrm{m}$. 
RA treatment at all concentrations (0.1-10 $\mu \mathrm{M})$ examined could induce the expression of phosphorylated NF-M (NF-M) proteins in NT2 aggregates. However, monolayer NT2 cells expressed phosphorylated NF-M only at higher concentrations of RA (1 or 10 $\mu \mathrm{M})$. The expression level of phosphorylated NF-M proteins in NT2 aggregates was elevated with increased concentration of RA used. A comparable expression level of phosphorylated NF-M proteins was detected when NT2 aggregates and monolayer NT2 cells were treated with 0.1 and $10 \mu \mathrm{M}$ RA, respectively (Figure 5, upper panel). This finding suggests that cell aggregation promotes the necessary biochemical changes that are important for the

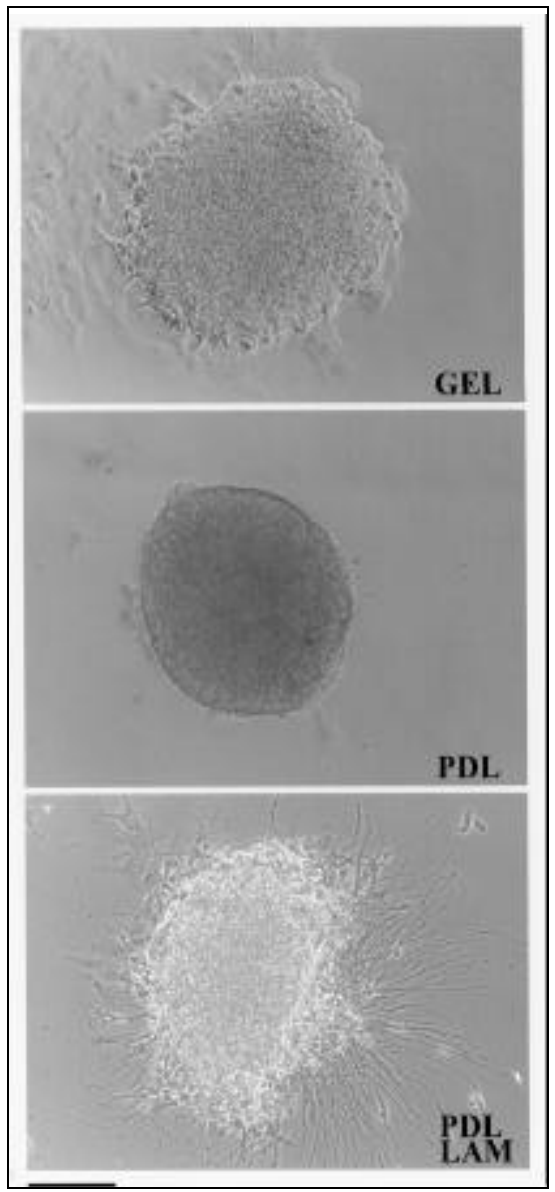

Figure 4. Effect of substratum on the neuronal differentiation of NT2 cells. NT2 aggregates were treated with RA $(10 \mu \mathrm{M})$ for 14 days and replated onto 24-well plates precoated with either PDL, LAM or a combination of both. The cell aggregates were cultured in RA-free medium for 2 days. The bright-field, phase-contrast photomicrographs are shown. Scale bar, $500 \mu \mathrm{m}$. neuronal differentiation of NT2 cells and therefore allows RA to induce neurofilament protein expression even at reduced concentrations.

Similar results were obtained when the expression of synapsin I proteins in RA-treated NT2 aggregates were examined (Figure 5, lower panel). A high level of synapsin I protein expression was detected in RA-treated (0.1-10 $\mu \mathrm{M})$ NT2 aggregates, but not in monolayer NT2 cell cultures. Note that the number of neurons that could be prepared diminished with a reduced concentration of RA, i.e., only a few neurons could be observed at $1 \mu \mathrm{M}$ RA, while almost no neurons were observed when the RA concentration was further reduced to $0.1 \mu \mathrm{M}$ (data not shown). In the absence of RA treatment, aggregation alone could not result in any observable neuronal phenotype.

\section{Short-Term RA Treatment is Enough for Neuronal Differentiation of NT2 by Aggregation}

Under monolayer culture conditions, a brief period of RA treatment resulted in changes in cell morphology without the formation of NT2 neurons. Therefore, continuous RA treatment of NT2 cells is essential for the neuronal differentiation of NT2 cells (1). It is possible that the sustained requirement of RA for the neuronal differentiation of NT2 is indeed due to the lack of cell/cell interaction. To examine this possibility, NT2 cells were maintained as aggregates in a bacteriological culture dish for 14 days, and RA treatment was given only during the first few days of aggregation (1-5 days). Neurons were observed following only 2-3 days of RA treatment, provided that the NT2 cells were maintained as aggregates for 14 days (Figure 6).

\section{DISCUSSION}

NT2 cells have been extensively used as in vitro model systems for various studies related to cancer therapy, CNS development and neurodegenerative diseases. However, the strategy used for the preparation of these NT2 neurons required lengthy RA treatment and might result in the loss of specific 
subpopulations of neurons. We aimed at developing a rapid approach to produce NT2 neurons by introducing other extrinsic factors, such as cell/cell interaction, that are essential during the process of neuronal differentiation. In the present study, we report that when NT2 cells were treated with RA while growing as cell aggregates in bacteriological plates, neurons were formed within 16 days. Using the standard protocol for differentiation, neuron-like cells could only be observed approximately five weeks after the RA treatment (17). In our study, the promotion of neuronal differentiation by cell aggregation is also revealed by the higher level of expression of phosphorylated NF-M proteins and the early expression of the synapsin proteins in RA-treated NT2 aggregates.

While cell aggregation can promote neuronal differentiation of NT2 cells, our findings suggest that it could not replace RA as an extrinsic factor to trigger the process of neuronal differentiation. In the absence of RA, there was no observable neuronal phenotype in NT2 aggregates, despite a very low level of phosphorylated NF-M expression. A low concentration of RA $(0.1 \mu \mathrm{M})$ was enough to induce the expression of phosphorylated NF-M in NT2 aggregates, but did not result in cells that resembled neurons. Thus, under cell aggregation conditions, lower concentration of RA is enough to induce neuron-related biochemical changes of the NT2 cells; however, not enough for the presentation of the neuronal phenotypes. Taken together, our findings suggest that RA serves as a decisive signal that initiate the biochemical changes involved in the neuronal differentiation process, such as the expression of a threshold level of neurofilament proteins. However, the presentation of the neuronal phenotypes is facilitated by the other extrinsic factors, such as the cell/cell interaction provided by the cell aggregation in this study.

Note that the period ( $\geq 14$ days) required for the maintainance of NT2 cells as aggregates is critical. This important experimental paradigm might well account for the inability of a previous study on NT2 cells to demonstrate any significant effects of cell aggregation on the formation of neurons (1). In our study, we have demonstrated that, provided the cell aggregation process was long enough ( $\geq 14$ days), RA treatment could be reduced (3-5 days). It is possible that RA serves to activate the pathways leading to the expression of the genes necessary for the neuronal differentiation, and that the maintenance of such expression is regulated either by the presence of RA or by the "factors" produced as a consequence of

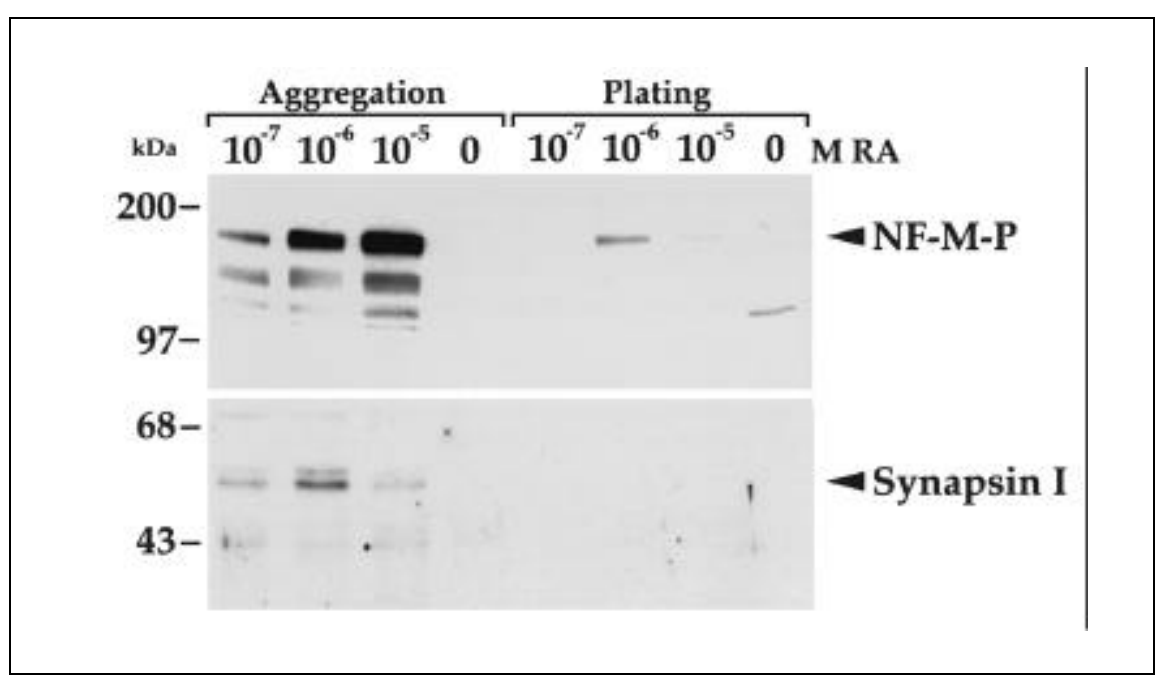

Figure 5. Expression of neuronal markers in RA-differentiated NT2 aggregates. NT2 cells were preaggregated by RA at concentration $\left(10^{-7}-10^{-5} \mathrm{M}\right)$ for 14 days and replated onto PDL/LAM-coated plates for two days. As control experiments, NT2 cells were plated at $2 \times 10^{5}$ cells $/ \mathrm{mL}$ as monolayer cultures and differentiated with RA $\left(10^{-7}-10^{-5} \mathrm{M}\right)$ for a total of 16 days. Total proteins were extracted, and western blot analysis was performed using antibodies specific for phosphorylated NF-M (upper panel) and synapsin I (lower panel). cell aggregation. Candidates for the genes regulated by RA include the neurotrophin receptors and their cognate ligands and novel sequences $(5,6)$.

The molecular mechanism underlying the effects of aggregation remains to be elucidated. It is possible that aggregation mimics the anti-proliferative effect of RA during the neuronal differentiation of NT2 cells, similar to what was previously demonstrated in immortalized neuroectodermal precursor cells (19). Alternatively, aggregation might induce the synthesis of RA in situ, thereby reducing the time required for RA treatment. Studies are in progress in our laboratory to elucidate the mechanism involved in the actions of aggregation during the RA-induced neuronal differentiation of NT2 cells. One useful

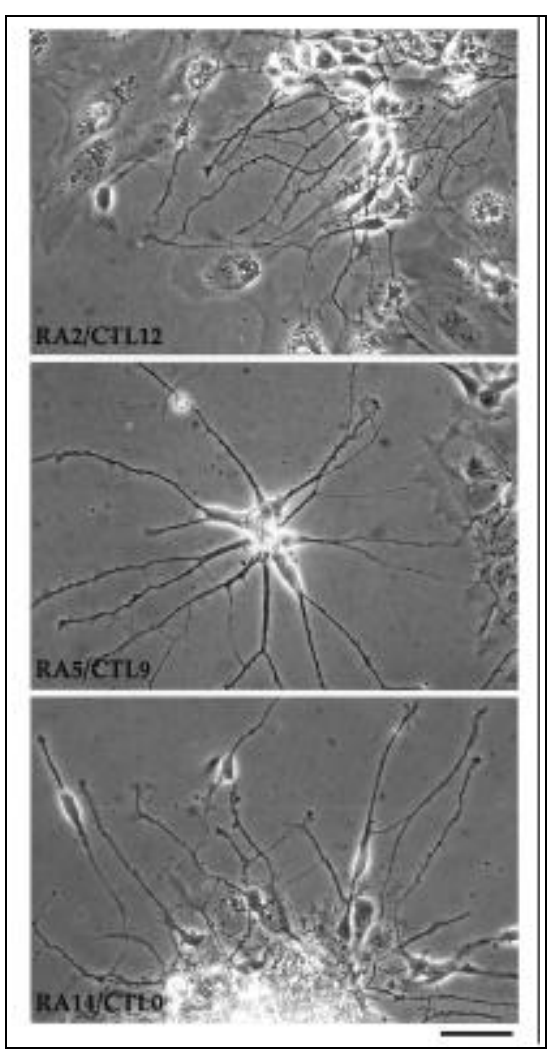

Figure 6. Aggregation reduced the duration of RA treatment required for the neuronal differentiation of NT2 cells. NT2 cells were treated as aggregates with RA $(10 \mu \mathrm{M})$ for either 2,5 or 14 days (RA2, RA5 and RA14, respectively) followed by incubation in RA-free medium as aggregates for the remaining period (CTL12, CTL9 and CTLO, respectively). The cell aggregates were plated onto PDL/LAM and further cultured for 2 days in the absence of RA. The bright-field, phase-contrast photomicrographs are shown. Scale bar, $50 \mu \mathrm{m}$. 
strategy is to perform extensive transcripts analysis to isolate candidate genes that might be involved in the aggregation process. This type of approach has proven to be useful in the P19 system (15). Further studies are needed to provide a better understanding on the role of aggregation in promoting neuronal differentiation of NT2 cells and on the interaction between extrinsic factors that influence the ultimate cell fate of a neuronal precursor cell.

NT2 neurons have been used extensively in studies aimed at understanding the mechanisms that underlie neuronal degenerative diseases such as Alzheimer's disease. For example, expression of specific forms of acetycholinesterase in NT2 cells allows the elucidation of the roles of these enzymes that are selectively lost in Alzheimer's patients (14). Moreover, NT2 neurons provide a rich source of neurons for use in cell transplantation therapy, which has recently been suggested to be one of the most promising treatment strategies for various neurodegnerative diseases (9). Transplantation of neurons into the CNS-lesioned site has resulted in the restoration of the normal nigrostriatal pathway in patients suffering from Parkinson's disease (20). Thus, the rapid protocol described in the present study will be useful in providing an efficient way to produce large quantities of neurons for basic research and for applications in cell therapy. Moreover, these transfectable postmitotic CNS neurons can also be used to deliver gene products directly into specific areas of the diseased human CNS.

\section{ACKNOWLEDGMENTS}

This study is supported by the Biotechnology Research Institute of Hong Kong University of Science and Technology (HKUST) and the ITDC Industry Support Fund (AF/142/96), Hong Kong.

\section{REFERENCES}

1.Andrews, P.W. 1984. Retinoic acid induces neuronal differentiation of a cloned human embryonal carcinoma cell line in vitro. Dev. Biol. 103:285-293.

2.Andrews, P.W., I. Damjanov, D. Simon, G.S. Banting, C. Carlin, N.C. Dracopoli and J. Fogh. 1984. Pluripotent embryonal carcinoma clones derived from the human teratocarcinoma cell line Tera-2. Lab. Invest. 50:147-162.

3.Bain, G., D. Kitchens, M. Yao, J.E. Huettner and D.I. Gottlieb. 1995. Embryonic stem cells express neuronal properties in vitro. Dev. Biol. 168:342-357.

4.Chambon, P. 1996. A decade of molecular biology of retinoic acid receptors. FASEB J. 10:940-954.

5.Cheung W.M.W., A.H. Chu and N.Y. Ip. 1997. Identification of candidate genes induced by retinoic acid in embryonal carcinoma cells. J. Neurochem. 68:1882-1888.

6.Cheung, W.M.W., A.H. Chu, M.-F. Leung and N.Y. Ip. 1996. Induction of trk receptors by retinoic acid in a human embryonal carcinoma cell line. Neuroreport 7:1204-1208.

7.Chyung, A.S.C., B.D. Greenberg, D.G. Cook, R.W. Doms and V.M.-Y. Lee. 1997. Novel beta-secretase cleavage of beta-amyloid precursor protein in the endoplasmic reticulum/intermediate compartment of NT2/N cells. J. Cell Biol. 138:671-680.

8.De Luca, L.M. 1991. Retinoids and their receptors in differentiation, embryogenesis, and neoplasia. FASEB J. 5:2924-2933.

9.Hoffer, B. and L. Olson. 1997. Treatment strategies for neurodegenerative diseases based on trophic factors and cell transplantation techniques. J. Neural Transm. Suppl. 49:1-10.

10.Iacovitti, L. and N.D. Stull. 1997. Expression of tyrosine hydroxylase in newly differentiated neurons from a human cell line (hNT). Neuroreport 8:1471-1474.

11.Jones-Villeneuve, E.M., M.W. McBurney, K.A. Rogers and V.I. Kalnins. 1982. Retinoic acid induces embryonal carcinoma cells to differentiate into neurons and glial cells. J. Cell Biol. 94:253-262.

12.Jones-Villeneuve, E.M., M.A. Rudnicki, J.F. Harris and M.W. McBurney. 1983. Retinoic acid-induced neural differentiation of embryonal carcinoma cells. Mol. Cell Biol. 3:2271-2279.

13.Larue, L., C. Antos, S. Butz, O. Huber, V. Delmas, M. Dominis and R. Kemler. 1996. A role for cadherins in tissue formation. Development 122:3185-3194.

14.Llanes, C., R.G. Collman, R. Hrin and D.L. Kolson. 1995. Acetylcholinesterase expression in NTera2 human neuronal cells: a model for developmental expression in the nervous system. J. Neurosci. Res. 42:791-802.

15.Marazzi, G., Y. Wang and D. Sassoon. 1997. Msx2 is a transcriptional regulator in the BMP-mediated programmed cell death pathway. Dev. Biol. 186:127-138.

16.Pleasure, S.J. and V.M.-Y. Lee. 1993. NTera 2 cells: a human cell line which displays characteristics expected of a human committed neuronal progenitor cell. J. Neurosci. Res. 35:585-602.

17.Pleasure, S.J., C. Page and V.M.-Y. Lee. 1992. Pure, postmitotic, polarized human neurons derived from NTera 2 cells provide a system for expressing exogenous proteins in terminally differentiated neurons. J. Neurosci. 12:1802-1815.

18.Smith, S.C., K.R. Reuhl, J. Craig and M.W. McBurney. 1987. The role of aggregation in embryonal carcinoma cell differentiation. J. Cell. Physiol. 131:74-84.

19.Toth, M., T. Shenk and E. Madarasz. 1995 Differentiation is induced in three-dimensional cultures of brain cells immortalized by the LAP mammalian regulatory system. J. Neurosci. Res. 41:764-774.

20.Trojanowski, J.Q., S.R. Kleppner, R.S. Hartley, M. Miyazono, N.W. Fraser, S. Kesari and V.M.-Y. Lee. 1997. Transfectable and transplantable postmitotic human neurons: a potential "platform" for gene therapy of nervous system diseases. Exp. Neurol. 144:92-97.

Received 5 November 1998; accepted 22 January 1999.

Address correspondence to:

Dr. Nancy Y. Ip

Department of Biology and Biotechnology

Research Institute

Hong Kong University of Science and

Technology

Clear Water Bay

Hong Kong, China

Internet: boip@ust.hk 